\title{
326 - Present-mindedness in adaptation to living with young onset dementia
}

Charlotte Berry ${ }^{1}$, Jan Oyebode (presenter) ${ }^{2}$, Carol Martin ${ }^{1}$.

${ }^{1}$ Leeds Institute of Health Sciences, University of Leeds, UK

${ }^{2}$ Centre for Applied Dementia Studies, University of Bradford, UK

Introduction: Research into the experiences of those with a diagnosis of young onset dementia is extremely limited and the research that does exist tends to be loss orientated.

Aim: This paper will focus on a doctoral study which aimed to explore the full spectrum of lived experience of those with young onset dementia, whilst considering the unique impact of diagnosis at a younger age and the possibility of personal growth.

Methods: Five individuals took part in semi-structured interviews within which they were invited to bring along artefacts that helped them to share their experiences. Transcribed interviews were analysed using Interpretative Phenomenological Analysis and superordinate and subordinate themes were identified.

Findings: Four superordinate themes emerged: 'Fear', 'Anger', 'Sadness', 'Contentment' with thirteen subordinate themes. The findings indicated that participants experienced feelings of fear and vulnerability in response to their diagnosis. Participants felt angry that they did not have a voice, not enough was being done for those with dementia and they were being stereotyped. Participants spoke of a depressive state of mind in which they grieved for their past self, experienced isolation and loneliness, and feelings of hopelessness and despair. Finally, participants spoke of a sense of contentment in relation to a preserved self, a sense of living alongside their dementia and a desire to live in the present; making the most of the here and now.

Conclusions: The findings highlight the transitory nature of participants' experiences as a result of multiple and repeated challenges to psychological equanimity. It suggested that these experiences could best be understood using lenses of lifespan development, bereavement and grief. The findings suggest that approaches to dementia care that focus on developing present-mindedness, acceptance and compassion may be particularly helpful. 